\title{
The Augusta Heart Study
}

\author{
Gaston Kapuku1, Santu Ghosh ${ }^{2}$, Vishal Doshi' ${ }^{1}$, Patricia Hall ${ }^{2}$, William Strong ${ }^{3}$, Frank Treiber ${ }^{4}$, David \\ Pollock $^{5}$, Jennifer Pollock ${ }^{5}$, Ryan Harris ${ }^{1}$, James Halbert ${ }^{1}$, Gregory Harshfield ${ }^{1}$, Varghese George ${ }^{2 *}$
}

${ }^{1}$ Georgia Prevention Institute, Department of Population Health Sciences, Medical College of Georgia, Augusta University, Augusta, GA, USA

${ }^{2}$ Division of Biostatistics and Data Science, Department of Population Health Sciences, Medical College of Georgia, Augusta University, Augusta, GA, USA

${ }^{3}$ Institute of Public and Preventive Health, Augusta University, Augusta, GA, USA

${ }^{4}$ College of Nursing, Medical University of South Carolina, Charleston, SC, USA

${ }^{5}$ Department of Medicine, University of Alabama at Birmingham, Birmingham, AL, USA

*Corresponding author: Varghese George, PhD, Department of Population Health Sciences, 1120 15th Street, AE-1005, Augusta, GA 30912, USA, Tel: 706-7210801; E-mail: vgeorge@augusta.edu

\begin{abstract}
Objective: The Augusta Heart Study is a NIH-funded multiracial longitudinal project, started in 1985, that examines cardiovascular health of children and adolescents in the Metro Augusta area. The objective was to develop a longitudinal cohort starting in childhood that would identify precursors and underlying pathophysiologic mechanisms that contribute to preclinical manifestations of cardiovascular disease (CVD). The focus was initially on sociodemographic factors, ethnicity, physical activity and obesity. In 2002, an additional focus on stress-related mechanisms of hypertension risk was initiated. The main objective of this paper is to introduce the rich database of this longitudinal multiracial cohort to researchers interested in assessing risk factors of lifetime development of CVD.

Methods: Overall, 585 youth were recruited during 1985-2002. This cohort was maintained, and new age-matching participants were added so that, by 2007, the participant pool included 300 African Americans and 307 European Americans.

Results: Valuable data, including childhood and young adulthood cardiovascular phenotypes and genotypes, were collected over a thirty-year span. Data were carefully curated and a comprehensive database was created to store data in digital format. As an illustration of the valuable research potential of the database, we performed a longitudinal analysis of the left ventricular mass using a mixed effect growth curve model.

Conclusions: The database allows longitudinal analysis of precursors of cardiovascular disease and their underlying mechanisms, allowing to explore beyond simple epidemiology and orienting toward translational research. This cohort may also be used to evaluate the development of other disease processes. Our analysis of the left ventricular mass points out to the early onset and rapid elevation of blood pressure and cardiac mass in African Americans compared to European Americans, and males compared to females.
\end{abstract}

Keywords: Biobehavioral stress; Cardiovascular disease; Chronic stress psychometrics; Essential hypertension; Left ventricular mass; Longitudinal cohort

\section{Introduction}

The Augusta Heart Study is a community-based multiracial longitudinal project, started in 1985, that examines cardiovascular health of children and young adults in the Augusta's Metropolitan Statistical Area (Metro Augusta). The Metro Augusta consists of the Richmond, Columbia, Burke, McDuffie and Lincoln counties in Georgia, and the Aiken and Edgefield counties in South Carolina, and it is part of the "stroke belt buckle". It is comprised of about 55\% European Americans (EA), 35\% African Americans (AA), 5.5\% Hispanics, and the remaining $4.5 \%$ of other races (Arensman, F. W., et al. 1989). This region has the highest incidence of mortality from stroke in the country. Seventy nine percent of the study participants came from Richmond, Columbia and Aiken counties.

Georgia and South Carolina are considered rural and lower income states, with a substantial portion of their populations living in poverty based on median household incomes. According to the 2013 statistics from the Census Bureau, about
Received date: December 7, 2018 Accepted date: February 20, 2019 Published date: February 28,2019

Citation: Kapuku, G., et al. The Augusta Heart Study. (2019) J Environ Health Sci 5(1): 15-23.

Copyright: (C) 2019 George. This is an Open access article distributed under the terms of Creative Commons Attribution 4.0 International License. 
a fifth (18\%) of the Metro Augusta population of all ages lives in poverty. Only $59 \%$ of the total Metro Augusta population has private health insurance, including those with some form of supplemental public insurance (Coleman-Jensen, Gregory, \& Singh, 2014). Compared to the national levels, hypertension $(30 \%$ vs. $35 \%)$ and hyperlipidemia (34\% vs.36\%) are more prevalent in this region (Mensah, Mokdad, Ford, Greenlund, \& Croft, 2005). It goes without saying that studying the cardiovascular health of The Metro Augusta population provides a unique opportunity to dissect the pathogenesis of hypertension and mechanisms contributing to cardiovascular health disparity in rural America.

The Augusta Heart Study is conducted by the Georgia Prevention Institute (GPI) (Levy, Treiber, Strong, \& Musante, 1989), which is currently housed within the Department of Population Health Sciences in the Medical College of Georgia at Augusta University. The Augusta University Health System strives to meet community needs for care, prevention of cardiovascular diseases (CVD), and stroke in Metro Augusta. The study is funded by the National Institute of Heart, Lung and Blood Institute of the NIH through the R01 and P01 mechanisms. The main objective of this paper is to introduce the rich database of this longitudinal multiracial cohort to researchers interested in assessing risk factors of lifetime development of CVD.

\section{Objectives of the Longitudinal Cohort}

The initial goal of the project that started in 1985 by was to develop a longitudinal cohort to identify risk factors responsible for the ever-expanding prevalence of cardiovascular disease in children, with a focus on sociodemographic factors, nutrition, physical activity and obesity. This cohort was referred to as the Studies of Children's Activity \& Nutrition (SCAN) Cohort. In 1989, a second longitudinal cohort, named the Hypertension Cohort, was initiated with the NIH support to examine the precursors of cardiovascular diseases (e.g., essential hypertension) in youth, with a focus on ethnicity and stress. The main objective of the second study was to address precursors that contribute to preclinical manifestations of cardiovascular diseases. In 2002, these cohorts were combined to form a single cohort for a project in a Program Project Grant with the overarching focus on stress related mechanisms of hypertension risk. The ultimate goal of the Program Project Grant, which is still active in its third 5-year cycle, is to empower primary health care providers with the ability to identify youth and young adults who are at an increased risk of developing CVD. Overall, by combining the two cohorts, 585 youth were recruited during 1985-2002. This cohort was maintained, and new participants of same age as the ongoing cohort were recruited so that by 2007, the participant pool was made of 300 African Americans and 307 European Americans. The participants were in the age range of 3-18 at their first enrollment.

The main goal of establishing a longitudinal cohort was to identify the bio-behavioral antecedents of cardiovascular diseases starting in childhood where CVD originates. Emphasis has always been on the interaction between lifestyle behavior (e.g., stress, stress coping skills, physical activity, smoking and diet), biochemical/hormonal pathways (e.g., renin-angiotensin system, sympathetic nervous system, hypothalamic pituitary adrenal (HPA) axis, sodium handling, endothelial function, insulin sensitivity, natriuretic hormones, oxidative stress, inflam- mation and dyslipidemia) and genetics/epigenetic underpinnings (Arensman, Treiber, Gruber, \& Strong, 1989; Davis, Kapuku, Treiber, Kumar, \& Snieder, 2002; Dekkers, Snieder, van den Oord, \& Treiber, 2002; Harshfield et al., 2002; Kapuku, Treiber, \&Davis, 2002; Kapuku, Treiber, Hartley, \& Ludwig, 2004; Treiber et al., 2003; Treiber et al., 2000). The longitudinal design has allowed us to examine the changes in contribution of genetic and environment stress (lower SES, high personal stress and body habitus) to blood pressure, left ventricular mass and carotid artery intima-media thickening from childhood to young adulthood (Hao et al., 2016; Hao et al., 2017; Kapuku et al., 2008; Kapuku et al., 1999; Wang et al., 2009; Ye et al., 2016).

The specific aims of the early cohorts were initially geared toward evaluating the independent and interactive effects of ethnicity and family history of essential hypertension on children's hemodynamic responses to laboratory stressors including exercise stress testing, video game challenge, social competence interview and forehead cold stimulation. Specifically, during the first decade of the Augusta Heart Study, the goal was to determine whether children with family history of hypertension, particularly AA who have hyper-reactive reaction to stress, are prone to develop early pathophysiological markers of essential hypertension such as blood pressure elevation at rest and in response to stressors and increased cardiac mass. During the second decade, the aims evolved to ascertaining how chronic environmental stress (early life stress, family dysfunction, unfair treatment, low individual and neighborhood socio-economic status, discrimination, poor coping, emotion and stressful working conditions) and unfavorable genotypes, separately or in combination, affected blood pressure (BP) reactivity to acute stress and eventual development of essential hypertension (EH). Moving forward into the third decade of the study, the specific aims were to probe the HPA axis, and examine a potential mediating role of increased adiposity and oxidative stress on cardiovascular reactivity over a period of time spanning from early childhood to late adolescence/early adulthood.

The primary goals of this manuscript are two-fold. (i) to provide longitudinal characterization of the AA and EA cohorts in the Augusta Heart Study who have positive, verified family histories of EH and premature myocardial infarction (MI), and who have been evaluated annually over the last 30 years, starting in 1985; and (ii) to make the data available for researchers to further investigate the longitudinal data for further discoveries.

\section{Methods}

\section{Cohorts that make up the Augusta Heart Study}

The Augusta Heart Study is comprised of two separately established original cohorts that were later merged into one follow-up cohort. The first cohort was part of SCAN, which evaluated children's lifestyle behaviors in association with coronary heart disease. This cohort includes a subset of 106 children (mean age $6.9 \pm 0.73$ years), recruited based on positive family history of premature coronary heart disease. Given the very young age of this cohort sample and the associated young age of their parents (36.4 \pm 4.5 years), a positive family history was defined as having one or more biological parents or grandparents who suffered myocardial infarction before age of 55 years. One hundred and five individuals had negative history of premature MI. 
In the Hypertension cohort, children were recruited using the family health questionnaires obtained from a county-wide public school screening of children in grades $\mathrm{K}$ through 8 whose families were interested in health research. The purpose of the questionnaire was to assess the incidence of hypertension and coronary artery disease in biological parents, and maternal and paternal grandparents. All children were born in the US, and they were normotensive and free of any chronic disease. The reported family histories of study participants were verified by a physician. A participant was defined as having positive family history of hypertension if at least one biological parent and one grandparent had high blood pressure (Musante et al., 2000; Treiber et al., 1993; Treiber et al., 2001). A participant was defined as having a negative history of hypertension if neither biological parent nor any of the four grandparents had been diagnosed with hypertension.

To recruit participants into the Hypertension Cohort, we had access to a total of 13,850 family health histories, of which $65.5 \%$ had complete information. Among those with complete information, $57.5 \%$ were classified as having positive family history $(\mathrm{FH}+), 12.7 \%$ had negative family history $(\mathrm{FH}-)$, $1.4 \%$ had only at least one parent with $\mathrm{EH}$ but no grand-parent, and, $28.4 \%$ had no biological parent with $\mathrm{EH}$ but one or more grand-parents had EH. A total of 1,865 participants participated in a school blood pressure screening from which a random sample of $345 \mathrm{FH}+$ children, stratified by ethnicity, gender, and family history of hypertension, was recruited. There were also 30 FH- children, consisting of 15 each of EA and AA, who had no family history of hypertension.

\section{Data Base and Storage Infrastructure}

Valuable data on different broad categories (see below in the Results Section) were collected over the thirty-year span of the study. Data were carefully curated and a relational database was created to store data in digital format. Actual data is stored in SQL Server databases located on Augusta University IT infrastructure. A public web information portal was created at https:// www.AugustaHeartStudy.org for the Augusta Heart Study. This website provides information about the study, a data dictionary, data collection methods, and information on necessary steps for outside investigators to request data for ancillary analyses. A private data portal was created on Augusta University intranet to provide easier access to data by providing different data views and data extraction procedures to principal investigators and faculty associated with the study. This easier access to the data over the intranet will maximize the project's usefulness and provide more detailed understanding of the study.

\section{Data Description}

As part of the general protocol for each cohort investigation, extensive physical and laboratory evaluations were performed at baseline and annual follow-up visits to identify the presence of subclinical disease markers as well as established and potential CVD risk factors. Participants returned on an annual basis until 2011, and every 18 months interval thereafter, and completed a battery of laboratory evaluations, which included resting, stress and field hemodynamics; repetitive collection of blood, urine and saliva; comprehensive echocardiography; flow mediated dilation; carotid artery intima-media-thickening, psychosocial metrics; and genetics/epigenetics measures. Out of approximately 8,000 variables collected over the past 30 years, about 3,000 variables, which are considered most pertinent, were selected to be released to the public. The variables are cataloged by broad general categories, as outlined below:

\section{- Demographic/Anthropometrics}

-Height, weight, body mass index, hip, waist, hip/waist ratio, skin folds

\section{- Hemodynamics}

- Resting/reactive/ambulatory blood pressure, cardiac output, total peripheral resistance, pulse wave velocity.

\section{- Biomarkers}

- Plasma: Electrolytes, lipid profile, glucose, creatinine, urea, endothelin-1, cortisolcytokines (IL-1 $\beta$, IL2, IL4, IL-5, IL6, IL-7, IL8, IL-10, IL-12p70, IL-13, TNF- $\alpha$, granulocyte-monocyte colony-stimulating factor, interferon- $\gamma$ ), high sensitivity $\mathrm{C}$ reactive protein, markers of oxidative stress (8-hydroxydeoxyguanosin, 8-isoprostane, thiobarbituric acid reactive substances), cystatin $\mathrm{C}$.

- Saliva: Cortisol

- Urine: Electrolytes (sodium, potassium, calcium)

\section{- Ultrasound-derived measures}

- Cardiac mass, relative wall thickness, ejection fraction, midwall fractional shortening, mitral inflow, carotid intima media thickness, flow mediated dilation.

- Family Health Information

- Parental \& grandparental CVD history, marital status, family composition

\section{- Chronic Stress Psychometrics}

- Multi-dimensional indices of socioeconomic status (e.g., Hollingshead four-factor index of social status, neighborhood SES), family environment score, day-to-day perceived discrimination, unfairness, MacArthur score of subjective community social status, Cook- Medley hostility, Spielberger anger expression (anger in, anger out, anger control), Spielberger trait anxiety inventory, Beck depression inventory, Stanford seven day physical activity recall, resilience, John Henryism score, adverse childhood experiences.

\section{Results}

\section{Descriptive Data Summary}

The cohort is balanced with respect to race and gender (Table 1). There were no significant differences in education levels between the two races. EA parents were more likely to be married and AA parents were more likely to be singles. EA families had significantly higher Hollingshead scores and higher socio-economic status (SES) compared to AA families. While EA fathers had higher SES than AA fathers, the difference was not significant between EA and AA mothers. A significantly higher proportion of AAs indicated racial unfairness. There was no difference in resilience between the two races. AAs had higher blood pres- 
Citation: Kapuku., et al. The Augusta Heart Study. (2019) J Environ Health Sci 5(1): 15-23.

sure readings at rest, during acute stress and in the field.

Table 1: Race and gender distributions of the AHS Cohort

\begin{tabular}{|l|c|c|c|c|}
\hline \multirow{4}{*}{ Race } & & Frequency & Percentage & P-value \\
\cline { 2 - 5 } & EA & 373 & 53 & \multirow{2}{*}{0.15} \\
\cline { 2 - 5 } & AA & 335 & 47 & \\
\hline \multirow{4}{*}{ Gender } & & Frequency & Percentage & P-value \\
\cline { 2 - 4 } & Male & 350 & 49 & \multirow{2}{*}{0.76} \\
\cline { 2 - 4 } & Female & 358 & 51 & \\
\hline \multirow{3}{*}{ Race X Gender } & & EA & AA & P-value \\
\cline { 2 - 4 } & Male & 195 & 155 & \multirow{2}{*}{0.12} \\
\cline { 2 - 5 } & Female & 178 & 180 & \\
\hline
\end{tabular}

The means and the standard deviations of a few relevant demographic/anthropometric variables such as age, height, weight, BMI and waist circumference, are given in Table 2. The sample sizes were very small for the first two visits, hence they were not included. These longitudinal data permit the examination of different models, in which adiposity contributes to $\mathrm{CV}$ risk in youth and young adults.

Blood samples were taken at different visits to determine the possible contributions of biochemical factors to the cardiovascular risk. The biomarkers include cortisol, cystatin $\mathrm{C}$, epinephrine, glucose, lipid profile, norepinephrine, urinary sodium, 8-isoprostane and 8-hydroxy 2'-deoxyguanosine (8-OHdG). We found that cortisol was inversely correlated with the family SES index (Hollingshead's Four Factor Social Status Index). There were sex and ethnicity differences in stress-induced release of endothelin-1, showing AA males with higher levels. Oxidative stress markers (8-isoprostane, $8-\mathrm{OHdG}$ ) were related not only to blood pressure reactivity but also to blood pressure levels in the field. Finally, cardiac structure is significantly associated with the metabolic syndrome, specifically to insulin and fasting glucose levels.

\section{An Example of Data Analysis: Longitudinal Analysis of Left Ventricular Mass Growth}

As an illustration of the valuable research potential of the Augusta Heart Study database, we performed a longitudinal analysis of LVMASS using a mixed effect growth curve model. In the model, the fixed effects represent the mean of the trajectory pooling of all the individuals within the sample, and the random effects represent the variance of the individual trajectories around these group means.

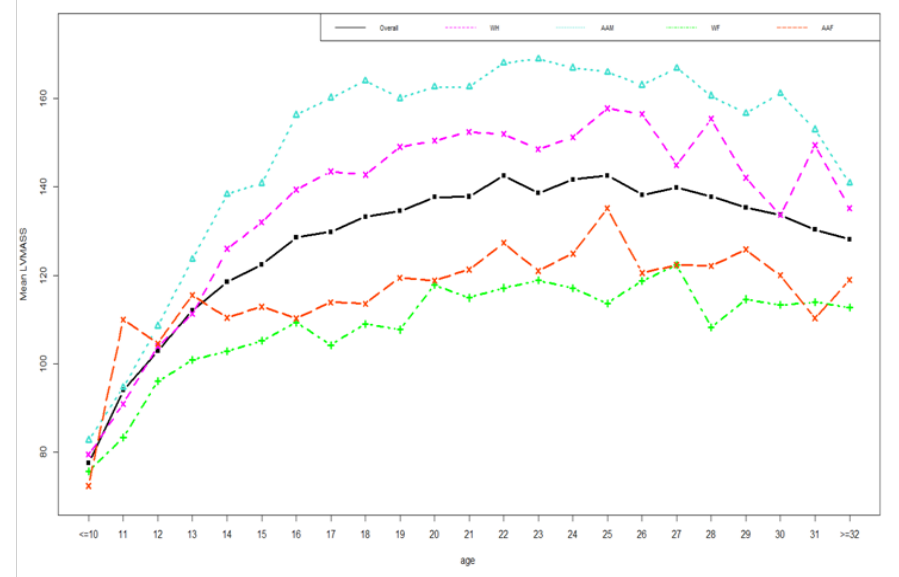

Figure 1: Mean LVMASS by age

Figure 1 illustrates the increase in mean LVMASS over time for the overall population as well as for the subgroups subgroups EA males, AA males, EA females, AA females. There may also be a quadratic relationship between age and LVMASS, especially with advancing age. These data suggest that a model to predict LVMASS should include both linear and quadratic fixed effects of age. The other fixed effects in the model for analysis include race, gender and height. It was also observed that the variability in LVMASS among participants at successive

Table 2: Descriptive summary of some relevant anthropometric variables in the cohort

\begin{tabular}{|c|c|c|c|c|c|c|}
\hline \multirow{2}{*}{ Visit } & \multirow{2}{*}{$\mathrm{N}$} & \multicolumn{5}{|c|}{ Mean \pm Standard Deviation } \\
\cline { 3 - 7 } & & Age (in years) & Height (in meters) & Weight (in kilograms) & BMI & Waist Circumference (in centimeters) \\
\hline 3 & 232 & $13.10 \pm 2.60$ & $1.55 \pm 0.14$ & $51.02 \pm 24.36$ & $22.49 \pm 6.40$ & $67.87 \pm 15.99$ \\
\hline 4 & 181 & $14.01 \pm 2.62$ & $1.59 \pm 0.12$ & $57.20 \pm 23.15$ & $23.59 \pm 6.82$ & $72.27 \pm 15.27$ \\
\hline 5 & 421 & $14.61 \pm 2.25$ & $1.63 \pm 0.10$ & $63.51 \pm 22.89$ & $22.34 \pm 5.15$ & $75.21 \pm 15.56$ \\
\hline 6 & 402 & $15.55 \pm 2.28$ & $1.66 \pm 0.09$ & $65.37 \pm 21.01$ & $22.74 \pm 5.06$ & $75.50 \pm 15.20$ \\
\hline 7 & 470 & $16.64 \pm 2.44$ & $1.68 \pm 0.09$ & $69.81 \pm 21.65$ & $24.21 \pm 6.14$ & $76.43 \pm 15.18$ \\
\hline 8 & 562 & $17.55 \pm 2.58$ & $1.69 \pm 0.09$ & $72.04 \pm 21.79$ & $25.02 \pm 6.66$ & $76.36 \pm 15.24$ \\
\hline 9 & 532 & $18.37 \pm 2.65$ & $1.70 \pm 0.09$ & $74.49 \pm 22.41$ & $25.15 \pm 6.23$ & $78.11 \pm 15.74$ \\
\hline 10 & 441 & $19.41 \pm 2.78$ & $1.71 \pm 0.09$ & $76.26 \pm 22.77$ & $25.61 \pm 6.28$ & $80.08 \pm 15.59$ \\
\hline 11 & 298 & $20.14 \pm 2.77$ & $1.71 \pm 0.10$ & $76.97 \pm 22.25$ & $24.94 \pm 6.31$ & $80.46 \pm 15.00$ \\
\hline 12 & 464 & $21.38 \pm 2.68$ & $1.71 \pm 0.09$ & $81.52 \pm 23.86$ & $25.40 \pm 6.17$ & $83.98 \pm 16.45$ \\
\hline 13 & 470 & $23.20 \pm 2.91$ & $1.71 \pm 0.09$ & $82.12 \pm 23.66$ & $27.17 \pm 7.12$ & $84.80 \pm 16.70$ \\
\hline 14 & 443 & $24.98 \pm 2.99$ & $1.71 \pm 0.09$ & $84.79 \pm 24.81$ & $27.98 \pm 7.52$ & $87.14 \pm 17.01$ \\
\hline 15 & 333 & $28.22 \pm 3.02$ & $1.71 \pm 0.09$ & $87.32 \pm 25.16$ & $27.85 \pm 7.94$ & $89.37 \pm 17.28$ \\
\hline 16 & 160 & $30.15 \pm 2.93$ & $1.71 \pm 0.09$ & $90.10 \pm 25.79$ & $29.47 \pm 8.18$ & $92.05 \pm 17.52$ \\
\hline
\end{tabular}


visits is not the same. To account for this variation in variability, we considered a model with random intercept and random slope. Thus, our growth curve model has the following form (L $=\mathrm{LVMASS}, \mathrm{G}=$ gender, $\mathrm{R}=$ race, $\mathrm{A}=$ age, $\mathrm{H}=$ Height):

$\mathrm{L}_{\mathrm{ij}}=\beta_{0}+\beta_{1} \mathrm{G}_{\mathrm{i}}+\beta_{2} \mathrm{R}_{\mathrm{i}}+\beta_{3} \mathrm{~A}_{\mathrm{ij}}+\beta_{3} \mathrm{~A}_{\mathrm{ij} 2}+\beta_{4} \mathrm{H}_{\mathrm{ij}}+\beta_{5} \mathrm{~A}_{\mathrm{ij}} * \mathrm{G}_{\mathrm{i}}+\mathrm{u}_{0 \mathrm{i}}+\mathrm{u}_{1 \mathrm{i}} \mathrm{A}_{\mathrm{ij}}$ $+\mathrm{u}_{2 \mathrm{i}} \mathrm{A}_{\mathrm{ij}}^{2}+\epsilon_{\mathrm{ij}}$

where $R_{i}$ and $G_{i}$ denote race and gender of the $i^{\text {th }}$ individual, and $\mathrm{L}_{\mathrm{ij}}, \mathrm{A}_{\mathrm{ij}}$ and $\mathrm{H}_{\mathrm{ij}}$ denote the LVMASS, age and height of the $\mathrm{i}^{\text {th }}$ individual at visit $\mathrm{j}$. Based on the evidence from the preliminary analysis, we included the interaction effect between age and gender in the final model, but not the interaction between age and race. We also included three random effects associated with each participant: a random intercept, a random age effect and a random age-squared effect. This allows each participant to have a unique parabolic trajectory with coefficients that vary randomly around the effects, defining the mean growth curve of LVMASS for each individual. The residuals $\epsilon_{\mathrm{ij}}$ in model (1) is assumed to be independent and identically distributed normal random variable with zero mean and unknown variance, conditional on a given participant. Further, we assume that the residuals and the random effects are independently distributed, which is a standard and reasonable assumption for a linear mixed effects model.

We assume that the random effects jointly follow a multivariate normal distribution with zero mean vector and unknown variance-covariance matrix. We use the restricted maximum likelihood method to estimate the variances and covariances of the three random effects $\mathrm{u}_{0 \mathrm{i}}, \mathrm{u}_{1 \mathrm{i}}$, and $\mathrm{u}_{2 \mathrm{i}}$. This variance-covariance matrix will be used to compute the test statistics for testing the linear and quadratic effects of age on LVMASS. To estimate the fixed effects parameters, we use the maximum likelihood estimation technique. We also checked the distribution assumption of model (1) by examining the distribution of the residuals and of the empirical best linear predictors for the random effects.

Table 3 displays the fixed-effects parameter estimates and the corresponding p-values. The main effect of gender on LVMASS is significant ( $p$-value $<0.001$ ) with the estimated mean LVMASS of 12.26 grams higher in males compared to females. The main effect of race on LVMASS is also significant (p-value $<0.001$ ) with an estimated mean LVMASS of 10.18 grams higher for AAs compared to whites. From Table 3, we also see that the interaction effect between gender and age on LVMASS is statistically significant (p-value $<0.001)$. The main effect of height on LVMASS is significant ( $p$-value $<0.001)$ with a positive correlation. Both the linear and quadratic terms of age are also significant predictors for LVMASS. The estimated coefficients of age and age (2) are positive and negative, respectively, with highly dominating linear coefficient, indicating that at early ages LVMASS increases with age and then it stabilizes as age increases.
Table 3: The fixed effects parameter estimates in the LVMASS analysis

\begin{tabular}{|l|l|l|l|l|}
\hline Parameter & Estimate & Standard Error & t-value & P-value \\
\hline intercept & -16.65 & 6.34 & -2.62 & 0.009 \\
\hline gender & 12.26 & 3.44 & 3.56 & $<.001$ \\
\hline race & -10.18 & 1.90 & -5.35 & $<.001$ \\
\hline age & 10.93 & 0.56 & 19.43 & $<.001$ \\
\hline age2 & -0.25 & 0.01 & -18.72 & $<.001$ \\
\hline height & 14.99 & 2.69 & 5.57 & $<.001$ \\
\hline genderXage & 0.92 & 0.17 & 5.56 & $<.001$ \\
\hline
\end{tabular}

Using the estimates of the fixed-effects parameters obtained by fitting model (1), the predicted mean LVMASS for given age, gender, race and height is given by

$\hat{\mathrm{L}}_{\mathrm{ij}}=-16.65+12.26 \mathrm{G}_{\mathrm{i}}-10.18 \mathrm{R}_{\mathrm{i}}+10.92 \mathrm{~A}_{\mathrm{ij}}-0.25 \mathrm{~A}_{\mathrm{ij}}{ }^{2}+14.99 \mathrm{H}_{\mathrm{ij}}$ $+0.92\left(\mathrm{~A}_{\mathrm{ij}}{ }^{*} \mathrm{G}_{\mathrm{i}}\right)$.

\section{Verification of Distributional Assumptions}

We checked the distributional assumptions for model (1), fitted using REML estimation, with graphical and statistical testing procedures. Figures 2 and 3 indicate that the assumption of normality for the residuals is acceptable. Further, we performed the Kolmogorov-Smirnov test of normality of residuals and the p-values was 0.07 . Hence, based on these results, the normality assumption of residuals seems to be reasonable and appropriate.

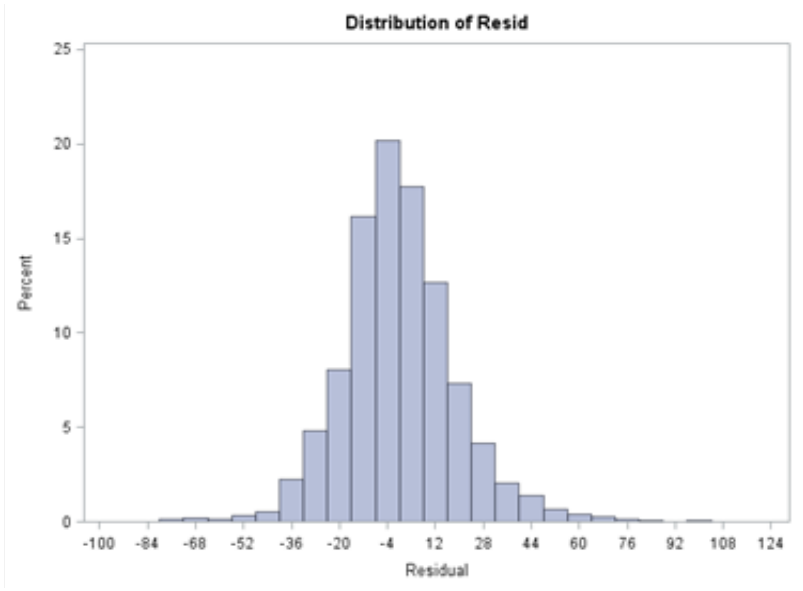

Figure 2: Residual histogram of the LVMASS analysis

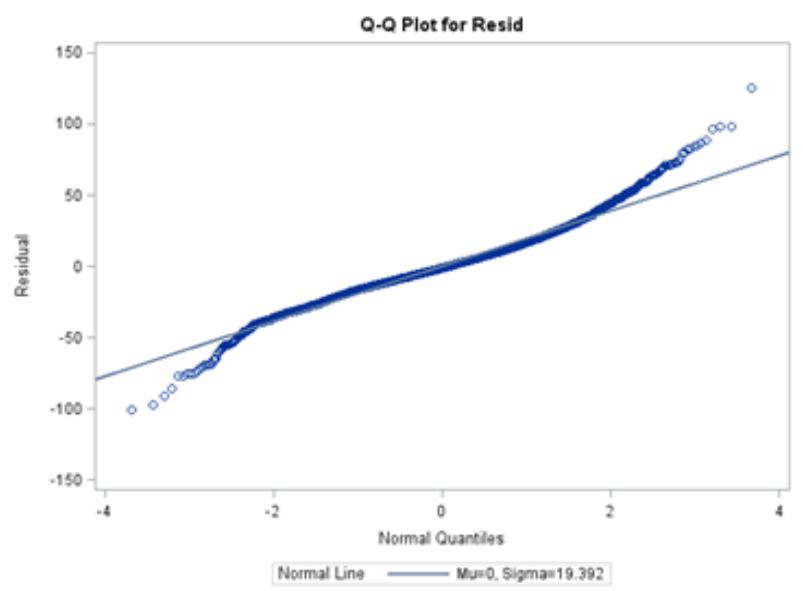

Figure 3: Normal Q-Q plot of residuals in the LVMASS analysis 
In order to test for the assumption of multivariate normality for the random effects, we applied the Royston's test, and the p-value was 0.09 . The Royston's multivariate normality test was based on empirical best linear unbiased predictors. The p-value suggests that a multivariate normal distribution assumption for the random effects is reasonable, which also implies that separately, each random effect component is normally distributed. Figures 4 and 5 show the histograms and the QQ plots for each random effect component.
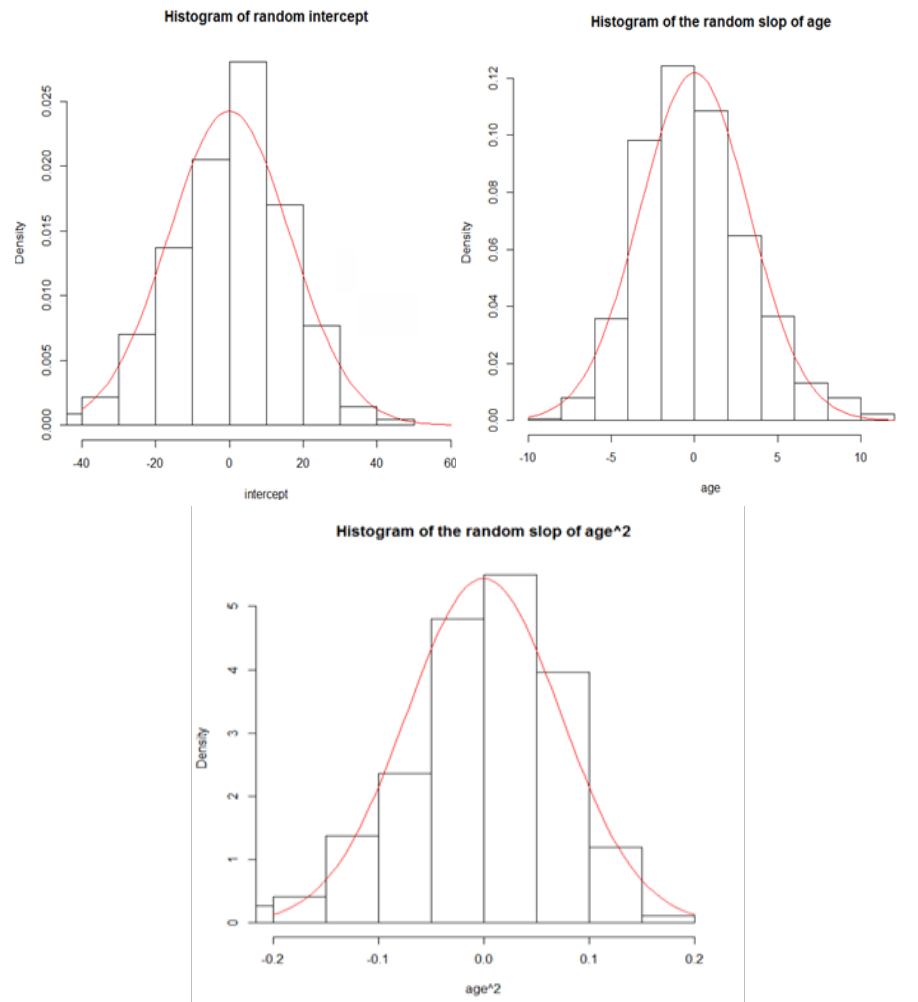

Figure 4: Histograms of the random effects in the LVMASS analysis.
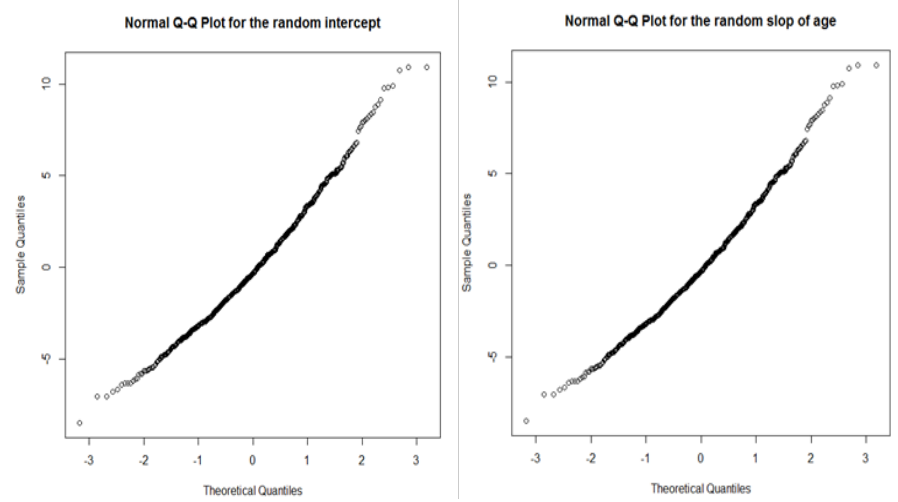

Figure 5: Normal Q-Q plots of the random effects in the LVMASS analysis

\section{Discussion}

The Augusta Heart Study represents a unique data base that can be used to understand the precursors of cardiovascular disease and their underlying mechanisms. Collectively, the Augusta Heart Study will help define the drivers of cardiovascular disease starting from the childhood through the young adulthood. For example, we consistently demonstrated that sex (males are at higher risk than females), race/ethnicity (AAs are at higher risk than whites), adiposity, positive family history of hypertension and premature myocardial infarction and increased cardiovascular stress responsiveness are predictive preclinical markers for CVD risk (Cook et al., 2001; Dekkers, Treiber, Kapuku, Van Den Oord, \& Snieder, 2002; Gregoski et al., 2013; Hao et al., 2016; Hao et al., 2016; Hao et al., 2017; Hao et al., 2018; Hartley, Treiber, Ludwig, \& Kapuku, 2004; Kapuku et al., 2017; Kapuku et al., 1999; Kapuku, Treiber, Hartley, \& Ludwig, 2004; Kapuku, Treiber, \& Davis, 2002; Mensah et al., 1999; Musante et al., 2000; Su et al., 2014; Treiber et al., 2001).

To reveal the preclinical CVD, we have evaluated the independent and interactive effects of ethnicity and family history of essential hypertension on children's hemodynamic responses to laboratory stressors including a video game challenge and forehead cold stimulation (Treiber, Kamarck, et al., 2003; Treiber et al., 2001). Our data points to the early onset and rapid elevation of blood pressure and cardiac mass in AA and males. These results can lead to further diagnostic and subsequent interventional protocols with the goal of primary prevention of essential hypertension and CVD. This would be of great benefit to reduce premature morbidity and mortality within population at high risk of developing CVD such as AAs.

Our findings from various analysis have been published and cited in the past (Dekkers, Treiber, Kapuku, \& Snieder, 2003; Dong, Wang, Zhu, Treiber, \& Snieder, 2004; Malhotra et al., 2004; Mensah et al., 1999; Musante et al., 2000; Poole, Snieder, Davis, \& Treiber, 2006; Treiber, Barbeau, et al., 2003; Treiber et al., 2000; Wang et al., 2006; Wright, Treiber, \& Davis, 1995; Wright, Treiber, Davis, Bunch, \& Strong, 1998; Zhu et al., 2008). We confirm our hypothesis that children with family history of hypertension, particularly AAs who have hyper-reactive reaction to stress, are prone to develop early pathophysiological markers of essential hypertension such as blood pressure elevation at rest and in response to stressors, increased cardiac mass, increased intima-media thickness, and elevated plasma endo- 
thelin-1 concentrations. These preclinical manifestations are accompanied by hormonal changes, suggesting their possible contribution as underlying mechanisms. Genetics and epigenetics underpinnings were also identified (Treiber et al., 2000;Dong, Harshfield, et al., 2004; Dong, Treiber, Zhu, Ludwig, \& Snieder, 2004; Snieder et al., 2002; Treiber, Barbeau, et al., 2003). These findings validate and/or expand those of other pediatric cohorts of cardiovascular disease including the Bogalusa Heart Study (Freedman, et al., 2007), Muscatine Study (Lauer \& Clarke, 1989), Cardiovascular Risk in Young Finns Study (Juhola, et al., 2013) and Childhood Determinants of Adult Health Study (Juonala, et al., 2011). Compared to the above cited studies, the Augusta Heart Study cohort has the advantage of being made of fifty percent of African American participants allowing the needed analysis of the role of ethnicity on cardiovascular manifestations.

The Augusta Heart Study cohort also represents a unique opportunity to tackle underlying mechanisms of cardiovascular disease and health disparities. Our findings permit ascertaining the differential contribution of specific risk factors and underlying mechanisms to cardiovascular disease manifestations. For example, we found significant gender and ethnicity differences in stress-induced release of endothelin-1(Treiber et al., 2000). The potentiating effect of environmental factors was also demonstrated by showing that carrier status of the $\mathrm{T}$ allele of the ET-1/Lys198Asn polymorphism showed greater diastolic blood pressure increases during the video game. Obese carriers, particularly those who were from lower socioeconomic status backgrounds exhibited the greatest increases in systolic blood pressure. (Treiber, Barbeau, et al., 2003; Treiber, Kapuku, Davis, Pollock, \& Pollock, 2002). We also demonstrated that impaired pressure natriuresis in response to acute stress may contribute to the development of essential hypertension and increasing cardiac mass, mostly in AAs.

The main strength of our cohort is the longitudinal design that permits researchers to identify CVD risk in groups that are underrepresented in medical research. Such information will lead to the development of more effective primary prevention programs involving lifestyle interventions in which the role of environmental stress will be taken into account (e.g. behavioral stress reduction, assertiveness/coping and /or parenting skills training), particularly for individuals at increased risk of CVD. Herein, we show case the cardiac mass growth by gender and ethnicity, yet, more host of other parameters contributing to cardiovascular risk can be longitudinally studied using our database. Other strengths include comprehensive data on ultrasound imaging of the heart and arteries, and a biorepository of blood, urine and saliva.

We only included AAs and EAs to reflect the majority of the Metro Augusta residents. An effort is undergoing to include the newly increasing populations in the region that also include Asian Americans and Hispanics.

\section{Conclusion}

The Augusta Heart Study is a community-based longitudinal study that examines the biological underlying mechanisms linking bio-behavioral stress to manifestations of cardiovascular diseases. The Study and its participants are making significant contributions to the promotion and detection of cardiovascular health. These contributions help advance the knowledge and awareness of blood pressure elevation and related cardiovascular disease risk in the Metro Augusta community and beyond.

The Augusta Heart Study represents a unique opportunity to assess lifetime development of CVD. The periodic evaluation of a multiracial cohort with a low attrition rate has great potential of generalization. This cohort may also be used to evaluate the development of other disease processes. Information on the criteria and the application process for accessing the data can be found at https://www.AugustaHeartStudy.org. We invite and strongly encourage interested researchers to explore this research opportunity to improve human health, by contacting us through our study website.

Acknowledgements: The authors acknowledge with gratitude Dr. Maurice Levy who cofounded the GPI with Dr. William Strong, Harry Davis for his past contributions in data analysis, and the dedicated GPI staff for the data collection and diligent maintenance of the cohort.

Conflicts of Interest: The authors report no conflicts of interest.

Funding Support: The current work is supported by the grant 5 P01 HL069999 15 from the National Heart, Lung and Blood Institute.

Financial Disclosure: The authors have no financial relationships relevant to this article to disclose.

Abbreviations: EA: European Americans; AA: African Americans; GPI: Georgia Prevention Institute; CVD: Cardiovascular Diseases; SCAN: Studies of Children's Activity \& Nutrition; HPA: Hypothalamic Pituitary Adrenal; EH: Essential Hypertension; MI: Myocardial Infarction; FH: Positive Family History; SES: Socio-Economic Status; LVMASS: Left Ventricular Mass;

\section{References}

- Arensman, F.W., Treiber, F.A., Gruber, M.P., et al. Exercise-induced differences in cardiac output, blood pressure, and systemic vascular resistance in a healthy biracial population of 10-year-old boys. (1989) Am J Dis Child 143(2): 212-216 PubMed | Crossref | Others

- Coleman-Jensen, A., Gregory, C., Singh, A. Household food security in the United States in 2013. (2014) PubMed |Crossref $\mid$ Others

- $\quad$ Cook, B.B., Treiber, F.A., Mensah, G., et al. Family history of hypertension and left ventricular mass in youth: possible mediating parameters. (2001) Am J Hypertens 14(4 Pt 1): 351-356.

PubMed | Crossref | Others

- Davis, C.L., Kapuku, G., Treiber, F.A., et al. Insulin resistance syndrome and left ventricular mass in healthy young people. (2002) Am J Med Sci 324(2): 72-75.

PubMed | Crossref | Others

- $\quad$ Dekkers, C., Treiber, F.A., Kapuku, G., et al. Growth of left ventricular mass in African American and European Ameri- 
can youth. (2002) Hypertension 39(5): 943-951.

PubMed | Crossref| Others

- $\quad$ Dekkers, J.C., Snieder, H., van den Oord, E.J., et al. Moderators of blood pressure development from childhood to adulthood: a 10-year longitudinal study. (2002) J Pediatr 141(6): 770-779.

PubMed | Crossref | Others

- $\quad$ Dekkers, J.C., Treiber, F.A., Kapuku, G., et al. Differential influence of family history of hypertension and premature myocardial infarction on systolic blood pressure and left ventricular mass trajectories in youth. (2003) Pediatrics 111(6 Pt 1): 1387-1393.

PubMed | Crossref | Others

- $\quad$ Dong, Y., Harshfield, G., Zhu, H., et al. The T594M sodium channel polymorphism and stress-induced sodium excretion in black youth: a gene by environment interaction model of hypertension risk. (2004) Am J Hypetens 17(5): 196A. PubMed | Crossref $\mid$ Others

- $\quad$ Dong, Y., Treiber, F., Zhu, H., et al. The sodium channel polymorphisms and stress-induced pressure natriuresis in black youth. (2004) Hypertension 44: 547.

PubMed | Crossref $\mid$ Others

- $\quad$ Dong, Y., Wang, X., Zhu, H., et al. Endothelin-1 gene and progression of blood pressure and left ventricular mass: longitudinal findings in youth. (2004) Hypertension 44(6): 884-890.

PubMed | Crossref | Others

- $\quad$ Freedman, D.S., Mei, Z., Srinivasan, S.R., et al. Cardiovascular risk factors and excess adiposity among overweight children and adolescents: the Bogalusa Heart Study. (2007) J Pediatr 150(1): 12-17. PubMed | Crossref | Others

- Gregoski, M.J., Buxbaum, S.G., Kapuku, G., et al. Interactive influences of ethnicity, endothelin-1 gene, and everyday discrimination upon nocturnal ambulatory blood pressure. (2013) Ann Behav Med 45(3): 377-386.

PubMed | Crossref | Others

- Hao, G., Wang, X., Treiber, F.A., et al. Growth of Carotid Intima-Media Thickness in Black and White Young Adults. (2016) J Am Heart Assoc 5(12): pii: e004147

PubMed | Crossref | Others

- Hao, G., Wang, X., Treiber, F.A., et al. Blood Pressure Trajectories From Childhood to Young Adulthood Associated With Cardiovascular Risk: Results From the 23-Year Longitudinal Georgia Stress and Heart Study. (2017) Hypertension 69(3): 435-442.

PubMed |Crossref | Others

- Hao, G., Wang, X., Treiber, F.A., et al. Body mass index trajectories in childhood is predictive of cardiovascular risk: results from the 23-year longitudinal Georgia Stress and Heart study. (2018) Int J Obes (Lond) 42(4): 923-925. PubMed | Crossref| Others

- Harshfield, G.A., Wilson, M.E., Hanevold, C., et al. Impaired stress-induced pressure natriuresis increases cardiovascular load in African American youths. (2002) Am J Hypertens 15(10 Pt 1): 903-906.

PubMed | Crossref | Others

- Hartley, B., Treiber, F., Ludwig, D., et al. Correlates of femoral artery flow mediated dilation in a multi-ethnic sample of 12- to 26-year-olds. (2004) Ethn Dis 14(2): 227-232.

PubMed | Crossref | Others

- Juhola, J., Magnussen, C.G., Berenson, G.S., et al. Combined effects of child and adult elevated blood pressure on subclinical atherosclerosis: the International Childhood Cardiovascular Cohort Consortium. (2013) Circulation 128: 217-224.

PubMed | Crossref | Others

- Juonala M., Magnussen C.G., Berenson G.S., et al. Childhood adiposity, adult adiposity, and cardiovascular risk factors. (2011) New Eng J Med 365: 1876-1885.

PubMed | Crossref | Others

- Kapuku, G., Treiber, F., Raouane, F., et al. Race/ethnicity determines the relationships between oxidative stress markers and blood pressure in individuals with high cardiovascular disease risk. (2017) J Hum Hypertens 31(1): 70-75. PubMed |Crossref| Others

- Kapuku, G.K., Ge, D., Vemulapalli, S., et al. Change of genetic determinants of left ventricular structure in adolescence: longitudinal evidence from the Georgia cardiovascular twin study. (2008) Am J Hypertens 21(7): 799-805. PubMed | Crossref | Others

- Kapuku, G.K., Treiber, F.A., Davis, H.C. Relationships among socioeconomic status, stress induced changes in cortisol, and blood pressure in African American males. (2002) Ann Behav Med 24(4): 320-325.

PubMed | Crossref | Others

- Kapuku, G.K., Treiber, F.A., Davis, H.C., et al. Hemodynamic function at rest, during acute stress, and in the field: predictors of cardiac structure and function 2 years later in youth. (1999) Hypertension 34(5): 1026-1031.

PubMed | Crossref | Others

- $\quad$ Kapuku, G.K., Treiber, F.A., Hartley, B., et al. Gender influences endothelial-dependent arterial dilatation via arterial size in youth. (2004) Am J Med Sci 327(6): 305-309.

PubMed | Crossref| Others

- $\quad$ Lauer, R. Clarke, W. Childhood risk factors for high adult blood pressure: the Muscatine Study. (1989) Pediatrics 84(4): 633-641.

PubMed | Crossref $\mid$ Others

- $\quad$ Levy, M., Treiber, F.A., Strong, W.B., et al. The Georgia Institute for the Prevention of Human Disease and Accidents. (1989) J Med Assoc Ga 78(10): 701-704.

PubMed | Crossref $\mid$ Others

- Malhotra, S., Poole, J., Davis, H., et al. Effects of NOS3 Glu298Asp polymorphism on hemodynamic reactivity to stress: influences of ethnicity and obesity. (2004) Hypertension 44(6): 866-871.

PubMed | Crossref | Others

- Mensah, G.A., Mokdad, A.H., Ford, E.S., et al. State of disparities in cardiovascular health in the United States. (2005) Circulation 111(10): 1233-1241.

PubMed | Crossref | Others

- $\quad$ Mensah, G.A., Treiber, F.A., Kapuku, G.K., et al. Patterns of body fat deposition in youth and their relation to left ventricular markers of adverse cardiovascular prognosis. (1999) Am J Cardiol 84(5): 583-588.

PubMed | Crossref | Others

- Musante, L., Treiber, F.A., Kapuku, G., et al. The effects of 
life events on cardiovascular reactivity to behavioral stressors as a function of socioeconomic status, ethnicity, and sex. (2000) Psychosom Med 62(6): 760-767.

PubMed | Crossref | Others

- $\quad$ Poole, J.C., Snieder, H., Davis, H.C., et al. Anger suppression and adiposity modulate association between ADRB2 haplotype and cardiovascular stress reactivity. (2006) Psychosom Med 68(2): 207-212.

PubMed | Crossref | Others

- Snieder, H., Harshfield, G.A., Barbeau, P., et al. Dissecting the genetic architecture of the cardiovascular and renal stress response. (2002) Biol Psychol 61(1-2): 73-95.

PubMed | Crossref | Others

- $\quad \mathrm{Su}$, S., Wang, X., Kapuku, G.K., et al. Adverse childhood experiences are associated with detrimental hemodynamics and elevated circulating endothelin- 1 in adolescents and young adults. (2014) Hypertension 64(1): 201-207.

PubMed | Crossref | Others

- $\quad$ Treiber, F.A., Barbeau, P., Harshfield, G., et al. Endothelin-1 gene Lys198Asn polymorphism and blood pressure reactivity. (2003) Hypertension 42(4): 494-499.

PubMed | Crossref | Others

- $\quad$ Treiber, F.A., Jackson, R.W., Davis, H., et al. Racial differences in endothelin-1 at rest and in response to acute stress in adolescent males. (2000) Hypertension 35(3): 722-725. PubMed | Crossref | Others

- $\quad$ Treiber, F.A., Kamarck, T., Schneiderman, N., et al. Cardiovascular reactivity and development of preclinical and clinical disease states. (2003) Psychosom Med 65(1): 46-62. PubMed | Crossref | Others

- $\quad$ Treiber, F.A., Kapuku, G.K., Davis, H., et al. Plasma endothelin-1 release during acute stress: role of ethnicity and sex. (2002) Psychosom Med 64(5): 707-713. PubMed | Crossref | Others

- $\quad$ Treiber, F.A., McCaffrey, F., Musante, L., et al. Ethnicity, family history of hypertension and patterns of hemodynamic reactivity in boys. (1993) Psychosom Med 55(1): 70-77. PubMed | Crossref $\mid$ Others
- $\quad$ Treiber, F.A., Musante, L., Kapuku, G., et al. Cardiovascular (CV) responsivity and recovery to acute stress and future $\mathrm{CV}$ functioning in youth with family histories of CV disease: a 4-year longitudinal study. (2001) Int J Psychophysiol 41(1): 65-74.

PubMed | Crossref | Others

- Wang, X., Ding, X., Su, S., et al. Genetic influences on daytime and night-time blood pressure: similarities and differences. (2009) J Hypertens 27(12): 2358-2364.

PubMed | Crossref | Others

- $\quad$ Wang, X., Poole, J.C., Treiber, F.A., et al. Ethnic and gender differences in ambulatory blood pressure trajectories: Results from a 15-year longitudinal study in youth and young adults. (2006) Circulation 114(25): 2780-2787.

PubMed | Crossref $\mid$ Others

- Wright, L.B., Treiber, F., Davis, H. John Henry coping style and CV reactivity in African American Youth: The influence of socioeconomic status (SES). (1995) Psychosom Med 57: 95.

PubMed | Crossref $\mid$ Others

- Wright, L.B., Treiber, F., Davis, H., et al. The role of maternal hostility and family environment upon cardiovascular functioning among youth two years later: socioeconomic and ethnic differences. (1998) Ethn Dis 8(3): 367-376.

PubMed | Crossref $\mid$ Others

- Ye, C., Pan, Y., Xu, X., et al. Pulse wave velocity in elastic and muscular arteries: tracking stability and association with anthropometric and hemodynamic measurements. (2016) Hypertens Res 39(11): 786-791.

PubMed | Crossref | Others

- Zhu, H., Yan, W., Ge, D., et al. Relationships of cardiovascular phenotypes with healthy weight, at risk of overweight, and overweight in US youths. (2008) Pediatrics 121(1): 115-122.

PubMed | Crossref | Others
Submit your manuscript to Ommega Publishers and we will help you at every step:

- We accept pre-submission inquiries

- Our selector tool helps you to find the most relevant journal

- We provide round the clock customer support

- Convenient online submission

- Thorough peer review

- Inclusion in all major indexing services

- Maximum visibility for your research

Submit your manuscript at

https://www.ommegaonline.org/submit-manuscript 